NASA Contractor Report 189110

AIAA-92-0418

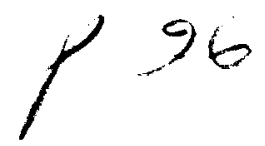

\title{
Development of an Analytical Method to Predict Helicopter Main Rotor Performance in Icing Conditions
}

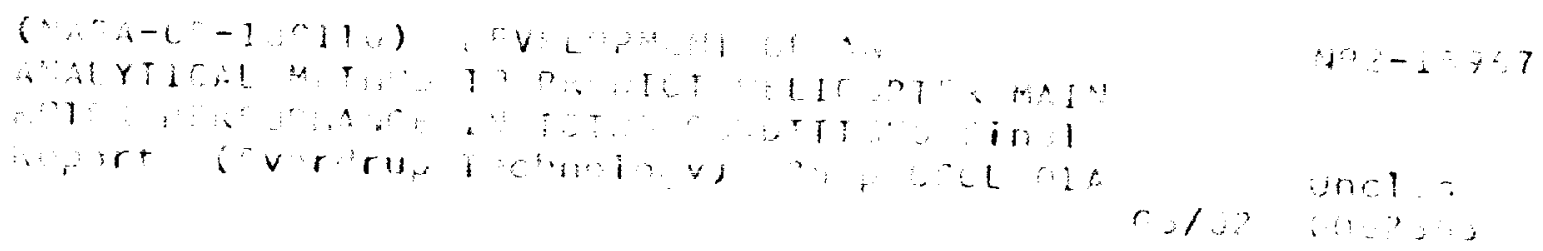

Randall K. Britton

Sverdrup Technology, Inc.

Lewis Research Center Group

Brook Park, Ohio

January 1992

Prepared for

Lewis Research Center

Under Contract NAS3-25266

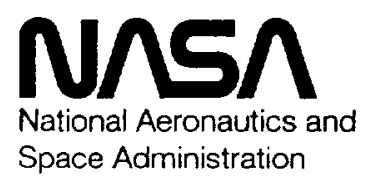


. 


\author{
Development of an Analytical Method to Predict \\ Helicopter Main Rotor Performance in Icing Conditions \\ Randall K. Britton \\ Sverdrup Technology, Inc. \\ Lewis Research Center Group \\ Brook Park, Ohio 44142
}

\begin{abstract}
Currently, an effort is being made by the NASA Lewis Research Center to develop an analytical procedure for calculating the performance degradation a helicopter experiences while operating in an icing encounter. A short discussion is given of the possibilities for performing such a calculation and reasons given for choosing the present approach. A complete description of the jobstream is given. Data taken from the NASA Lewis model rotor icing test program is used as a data base for comparison. Comparisons are also made between this method and the more traditional method based on empirical correlation. Conclusions are drawn as to how well results compare with experiment. Guidelines for calculation procedures are given. Limitations of this type of jobstream are pointed out and recommendations are made for future improvements.
\end{abstract}

\title{
Nomenclature
}

\begin{tabular}{|c|c|}
\hline A & Damping Length Constant \\
\hline$c_{i j}$ & Interaction Coefficient Matrix \\
\hline & Local Ice Thickness, m \\
\hline $\mathrm{E}_{\mathrm{m}}$ & Total Collection Efficiency \\
\hline f & Freezing Fraction \\
\hline $\mathbf{k}^{+}$ & Dimensionless Sand Grain Roughness \\
\hline $\mathbf{k}_{\mathbf{z}}$ & Equivalent Sand Grain Roughness, $\mathrm{m}$ \\
\hline L & Mixing Length \\
\hline LWC & Liquid Water Content, $\mathrm{g} / \mathrm{m}^{3}$ \\
\hline MVD & Volume Median Droplet Diameter, $\mu \mathrm{m}$ \\
\hline $\mathrm{m}_{\mathrm{c}}$ & Mass Flow Rate Due To Impinging Liquid, $\mathrm{kg} / \mathrm{sec}$ \\
\hline $\mathbf{m}_{\mathbf{i}}$ & Mass Flow Rate Which Freezes, kg/sec \\
\hline $\mathbf{m}_{\mathbf{r}}$ & Mass Flow Rate Due To Runback, kg/sec \\
\hline $\mathbf{R}$ & Rotor Blade Radius, m \\
\hline T, & Static Temperature, ${ }^{\circ} \mathrm{C}$ \\
\hline $\mathbf{u}^{\circ}$ & Inviscid Velocity, $\mathrm{m} / \mathrm{sec}$ \\
\hline$u_{c}$ & Tangential Edge Velocity, $\mathrm{m} / \mathrm{sec}$ \\
\hline$u_{p}$ & Total Normal Velocity Component, $\mathrm{m} / \mathrm{sec}$ \\
\hline $\mathrm{u}_{\mathrm{T}}$ & Total Tangential Velocity Component, $\mathrm{m} / \mathrm{sec}$ \\
\hline & Friction Velocity, $\mathrm{m} / \mathrm{sec}$ \\
\hline
\end{tabular}




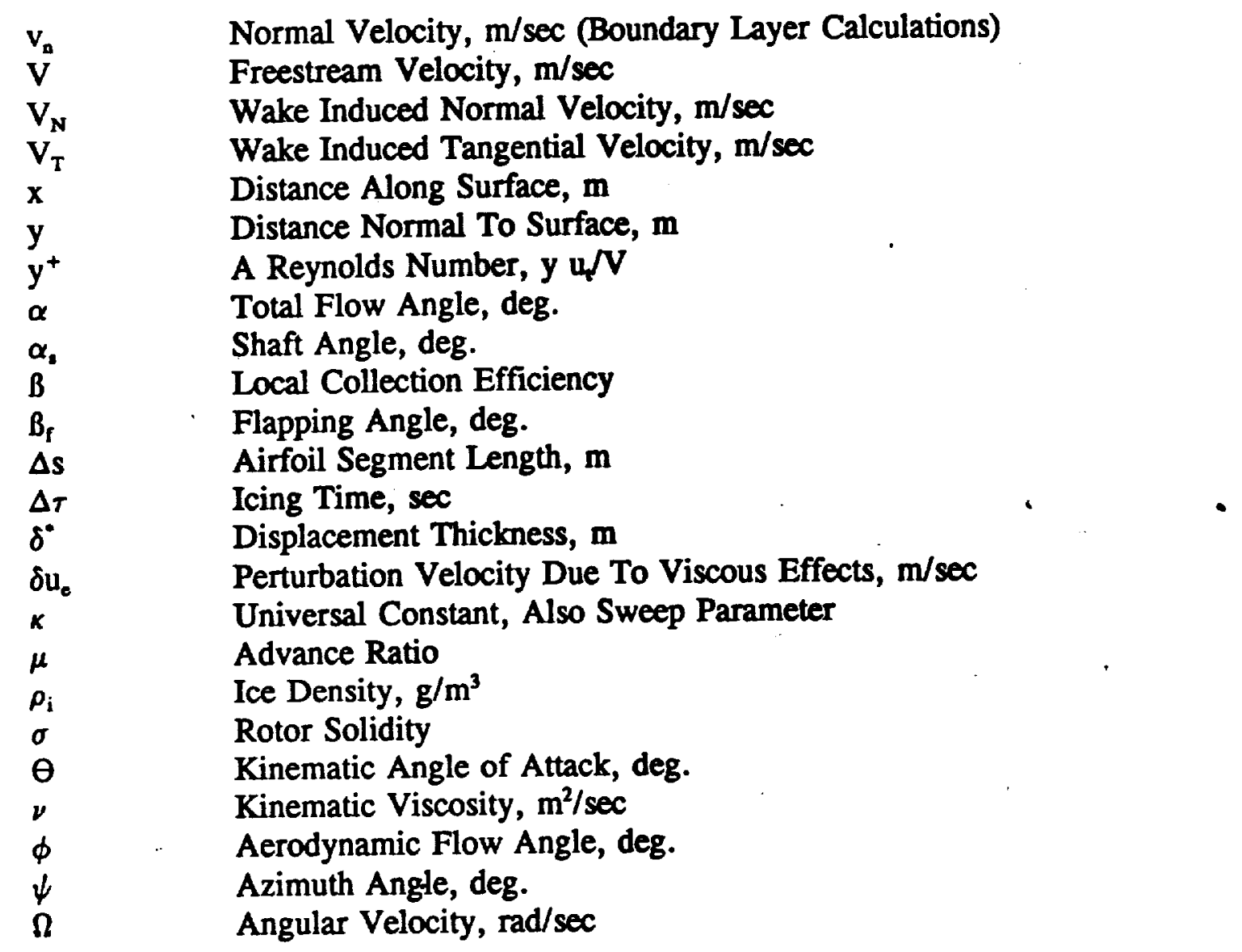

\section{Introduction}

Historically, certification of a helicopter for flight into known icing conditions has been a problem. This is because of the current emphasis on flight testing for verification of system performance. Flight testing in icing conditions is difficult because, in addition to being dangerous and expensive, many times conditions which are sought after cannot be readily found in nature. The problem is compounded for helicopters because of their small range in comparison to many fixed wing aircraft. Thus, helicopters are forced to wait for conditions to occur in a certain region rather than seeking them out. These and other drawbacks to flight testing have prompted interest in developing validated alternatives to flight testing. One such alternative is theoretical prediction. It is desirable to have the ability to predict how a helicopter will perform when subjected to icing conditions. Because theoretical prediction will never completely replace flight testing, the most logical approach is to have a program which blends flight testing, analytical prediction, and wind tunnel testing. A validated analytical approach would be invaluable for use in conditions which are difficult to find in nature. It should be noted that a theoretical analysis of the effects of icing on an unprotected rotor would still be valuable even though it is highly unlikely that any helicopter will be certified to fly into icing with no ice protection system. An accurate prediction would yield information which could be used for the design of ice protection systems, especially in terms of cycle times. This type of 
analysis would also provide knowledge of what would happen in the event of an ice protection system failure.

A complete analysis of the effects of an icing condition on the performance of a helicopter rotor includes prediction of the ice accretion, the aerodynamic penalties associated with the ice accretion, and ice shedding. The main thrust of this paper is the investigation of an analytical means of predicting the aerodynamic penalties associated with a given icing condition. In the past, aerodynamic penalties on the rotor have been predicted using an empirical relationship developed by Flemming. ${ }^{1}$ The correlation has shown reasonable agreement with experimental data taken in the NASA Lewis Icing Research Tunnel (IRT). The Flemming correlation also has the advantage of being very simple. However, because it is a correlation derived from an empirical set of data, it may have some limitations in terms of general application to full scale rotors. Recent developments in areas of efficiency and grid generation have made Navier-Stokes analysis a viable option for the calculation of performance of iced airfoils. ${ }^{2}$ However, in the present analysis, application of a Navier-Stokes code would be extremely unwieldy in terms of CPU time. This aspect will be discussed in more detail later. The Interactive Boundary Layer (IBL) analysis developed by Cebeci ${ }^{3}$ shows a great deal of promise. It has an advantage over the Navier-Stokes analysis in that it requires no grid and is not computationally intensive. Thus, the IBL scheme was selected for investigation in the current work.

\section{Method of Analysis}

Predicting the effects of an icing condition on the performance of a helicopter main rotor is a complicated task with several steps of calculation. A general outline of the required steps is given in flowchart form in Figure 1. Initially, the clean performance of the rotor is needed as a starting point. Then, the ice accretion along the radius of the rotor is calculated. A check is done to see where and when, if any, natural shedding has taken place. Once the new geometry of the iced rotor is known, aerodynamic performance coefficients are calculated. Then, finally the new performance of the overall rotor is determined based on this information. In the current analysis the various steps are performed by three major codes. Rotor performance is calculated by the lifting line method of B65, a code developed by Boeing Helicopters. Traditionally, lifting line theory has been the main vehicle for helicopter performance calculations and most helicopter companies have a code similar to B65. The ice accretion information is obtained by using the procedure of LEWICE', developed by the NASA Lewis Research Center. Finally, the IBL method is used to calculate the aerodynamic coefficients of the iced geometry. The natural shedding check is performed by using the empirical correlation developed by Flemming. ${ }^{1}$ For the purposes of this work, all shedding is assumed to be natural. Thus, no de-icing module is currently included.

\section{Calculation Procedure}

Because LEWICE and IBL are both two dimensional codes, a performance code like B65, which uses blade element theory makes for an easy transfer of information between the various codes. B65 relies heavily on experimental data tables for two dimensional lift, drag, and 
moment values which are integrated to obtain the overall rotor performance. Before an in depth discussion of the theory behind each of the various codes is given, a complete description of the run procedure of the jobstream is warranted.

The calculation begins by the user specifying the clean lift desired. This lift should correspond to the rotor lift experienced at the onset of icing. Once the clean solution is obtained, the stored values of Mach number and angle of attack for each radial and azimuthal station are passed to LEWICE. Here, the azimuthal values are averaged to obtain one value of Mach number and angle of attack for each radial station. This is done because LEWICE is a steady code which only requires one value of angle of attack and velocity for a given twodimensional airfoil section. It should be noted that a slightly different version of the traditional LEWICE code was used for these calculations. The version used is essentially the same except that it operates a more efficient form of the potential flow solver. ${ }^{3}$ Once the averaged values of Mach number and angle of attack are known, the ice shape is calculated at each radial station along the blade, based on input icing conditions. It is assumed here that symmetry exists between the blades of the rotor. The current approach uses 19 radial stations. This averaging technique is discussed in more detail in a later section. It should be noted that, before LEWICE makes any calculations, a check is done to see if any shedding takes place. If shedding does take place somewhere on the rotor, the icing time is modified appropriately for that radial station. For example, if the icing time is 60 seconds and it is found that a shed occurred at the $98 \%$ radial station at 40 seconds, then the new icing time for the $98 \%$ radial station is changed to 20 seconds. It is assumed that all sheds leave a clean surface with no residual ice. Once this part of the calculation is complete, the new iced airfoil geometries of each radial station are stored. While an averaging procedure is used for calculation of the ice shapes, no such procedure is employed for performance calculations. All changes in lift and drag due to icing are applied locally, that is, at each radial and azimuthal location. The two-dimensional "iced" airfoils are passed to the IBL procedure. The task for the IBL procedure is to calculate a new drag polar for each radial station separately. The reason that this is necessary is because of the way that B65 performs its calculations. B65 is an iterative code and thus varies the angle of attack in order to obtain a specified lift. Therefore, the performance characteristics of an airfoil section, whether clean or "iced", must be known for a range of Mach numbers and angles of attack in order for B65 to converge to a solution. This means that IBL must read in each "iced" radial station and then calculate its characteristics for a range of Mach numbers and angles of attack. This results in hundreds of loops through the IBL procedure for each jobstream run. This is a major reason why a Navier-Stokes type analysis would be unwieldy here. The current architecture of the jobstream would have to be reconsidered in order to make Navier-Stokes a viable option. The performance maps of each of the radial stations are stored in terms of deltas. The deltas are obtained by subtracting the clean values of lift and drag from the iced values. Currently, no modification of moment coefficient is made for icing. The final step then consists of calculating the iced performance with B65, using the modified airfoil tables. The following sections discuss the theory behind each of the codes in more detail.

\section{Helicopter Performance Prediction}

The core of the jobstream is the performance prediction code. Although, many other 
methods exist, it has been decided to use Boeing Helicopters' B65 performance code, which uses lifting line theory in its aerodynamic model. The code is proprietary in nature, and thus, detailed descriptions of the various models within the code will not be included here. Discussion will be limited to a brief description of lifting line theory in general.

Lifting line theory makes use of blade element theory in which the rotor blade is divided into several discrete radial sections. Characteristics are calculated for each section as a function of radial and azimuth location and then integrated to obtain rotor performance values. Each section acts as a steady 2-D airfoil section - with three dimensional and unsteady effects, including dynamic stall, usually included as corrections to the overall behavior. Section characteristics are normally obtained as a function of Mach number and angle of attack by use of an empirical set of data tables.

The crux of a lifting line performance code is the calculation of the flow angle of attack over a 2-D slice of the rotor blade. This flow angle is given by

$$
\alpha=\phi+\theta
$$

where $\phi$ arises from the purely aerodynamic behavior and $\theta$ comes from the blade aeroelasticity. The aeroelastic behavior of the rotor is usually significant because the high aspect ratio blades tend to be very flexible and respond to the various airloads. A typical velocity triangle for a rotor section is given in Figure 2. Here the total normal velocity component, $u_{p}$, and the tangential component, $u_{T}$, are given by

$$
\begin{aligned}
& u_{p}=V \cos \left(\alpha_{\rho}\right) \sin \psi+\frac{d \beta_{f}}{d t}+V_{N_{m}} \\
& u_{T}=\Omega R+V \sin \left(\alpha_{\rho}\right) \sin \psi+V_{T_{W}}
\end{aligned}
$$

In a normal calculation the rotational speed, $\mathrm{RR}$, and shaft angle, $\alpha_{\imath}$, are known. Thus, the remaining quantities which need to be calculated are the flapping velocity and the normal and tangential components of the wake induced velocity. These values are obtained through various procedures and iterated upon until the desired trimmed thrust level is achieved. A more complete description of blade element theory can be found in Reference 5 and an in depth discussion of a typical lifting line analysis can be found in Reference 6.

\section{Ice Accretion Prediction}

A numerical analysis LEWICE 4 has been developed by the NASA Lewis Research Center which has the ability to predict the analytical ice shape which accretes on a given component exposed to an icing condition for a known period of time. This analysis models four critical steps in the icing process, which are: 
(1) Flowfield calculation about the component;

(2) Water droplet impingement characteristics;

(3) Heat transfer processes;

(4) Ice accumulation normal to the surface.

Each of these areas are discussed briefly in the following sections.

\section{Flowfield Calculation}

Predicting the flowfield about a body which has an accreted ice shape presents several challenges because of the irregular effective airfoil shape which often occurs. The potential flow program developed by Hess and Smith ${ }^{7}$ is incorporated into LEWICE. This method makes use of distributed sources, sinks, and/or vortices to describe the flowfield about a body which has been modeled by a series of line segments. Comparisons of results to experimental clean body data have been favorable for the normal ranges of incompressible flow. A potential problem does exist for applying the assumption of incompressible flow to regions near the tip of a rotor blade. It is felt that the high centrifugal forces in this region will cause shedding to occur, especially for warmer temperatures. Thus, this particular limitation is not seen as causing any significant difficulty.

\section{Impingement Characteristics}

Of primary importance in any ice accretion analysis is characterization of the region of impinging water droplets. This characterization consists of the limits of impingement as well as the distribution of the mass of the impinging liquid. This is typically obtained by performing an analysis of droplet trajectories from far upstream. Two important parameters which arise are the total and local collection efficiency. The total collection efficiency is defined as the ratio of the total mass of impinging liquid over the theoretical mass of impinging liquid which would occur if all of the droplet trajectories were straight lines. The local collection efficiency is based on the same definition, except that it pertains to a specific location on the body. A pictorial representation of this is given in Figure 3.

The droplet trajectory analysis used in LEWICE is based on the work of Frost, Chang, Shieh, and Kimble. ${ }^{8}$ The method has the ability to calculate trajectories and impingement characteristics of an arbitrarily shaped particle. Although generality has been maintained, for this application it can usually be assumed that the particles are spherical and gravity forces are negligible. This simplifies the calculation somewhat.

\section{Heat Transfer Processes}

The freezing process is modeled in LEWICE by performing a mass and energy balance on a control volume located on the surface and extending beyond the boundary layer, as shown in Figure 4. Each segment which describes the surface of the accreting component has a corresponding control volume. The runback model first developed by Messinger' is incorporated here. An important quantity in this analysis is the freezing fraction which is the ratio of freezing liquid within a control volume over the total amount of liquid entering. The freezing fraction 
can be expressed as:

$$
f=\frac{m_{i}}{m_{c}+m_{r}}
$$

Once the temperature and the freezing fraction are known, the mass balance calculates the mass flow of the liquid runback out of the control volume. Any liquid which leaves the control volume is assumed to leave in the direction away from the stagnation point. Surface roughness strongly influences the local heat transfer processes. The equivalent roughness concept is used here. This concept models the actual surface roughness by using an average value which yields the same heat transfer characteristics. This aspect of the analysis is considered a weak point and much effort is being expended to improve the current heat transfer model in general. ${ }^{10}$

\section{Surface Ice Growth}

The ice is assumed to grow normal to the surface. The ice growth rate can be defined using the expression for the freezing fraction and is given as:

$$
m_{l}=f\left(m_{c}+m_{p}\right)
$$

This can be redefined to yield an expression for the ice thickness as:

$$
d_{i}=\frac{m_{i} \Delta \tau \Delta s}{P_{i}}
$$

The iced component is then calculated by adding the corresponding ice thickness normal to each matching segment. This results in a new airfoil surface coinciding with the specified icing time.

\section{Ice Accretion Calculation Procedure}

Using LEWICE to predict a 2-D ice accretion at a specified radial location on a helicopter rotor is not a straightforward calculation. Thus, a brief discussion of the procedure used in this calculation is warranted. LEWICE is a steady state code designed for use on a fixed wing where the velocity and angle of attack are constant throughout a given time step. However, for application to a helicopter in forward flight where the local angle of attack and Mach number are constantly changing some averaging procedure is necessary. A technique developed in 1983 by Korkan, Dadone, and Shaw ${ }^{11}$ dealt with this problem. Here, while attempting to simplify the analysis of a helicopter main rotor in forward flight with a rime ice accretion, several methods 
of averaging were investigated. It was found that if the local Mach number and angle of attack produced by a helicopter performance code were averaged and input into an icing performance degradation analysis the predicted change only differed by $\pm 2 \%$ over that of the traditional method of calculating values at specific azimuth locations around the disk. In view of this, a similar averaging technique is employed in the present study. First, trimmed performance values are calculated using a helicopter performance code. The local flow angle at the desired radial location required for trim is then averaged azimuthally. The local velocity is taken to be the rotational velocity at the specified radial location. This is, in effect, the averaged velocity. These values are then used in LEWICE as the velocity and angle of attack. The calculation procedure from then on is carried out as any normal LEWICE calculation using established guidelines given in the LEWICE User's Manual." As shown in Figures 5 and 6, previous comparisons of results obtained using this procedure to experiment have been very good. More in depth comparisons using this procedure are given in Reference 12. .

\section{Performance Penalty Prediction}

Critical to predicting the performance degradation of an icing encounter on an aircraft is the ability to accurately compute the associated changes in sectional lift, drag, and moment characteristics. Navier-Stokes methods have this ability. ${ }^{2}$ However, current Navier-Stokes schemes require a significant amount of computer time. A great deal of research is being performed in this area and as mainframe technology advances and the efficiency of the schemes improve it is anticipated that Navier-Stokes methods will eventually be the means for this type of calculation. However, -at the present, a simpler short term solution is needed. The Interactive Boundary Layer (IBL) procedure developed by Cebeci ${ }^{3}$ has been chosen for its simplicity and apparent success at predicting drag values. The IBL procedure is also attractive in that it does not require a computational grid.

The IBL approach consists of solution of inviscid flow and boundary layer equations which are coupled so that one influences the other. The inviscid calculations are derived from the panel method developed by Hess and Smith. ${ }^{7}$ This method models the airfoil and ice shape by a series of line segments which contain distributed source and/or vorticity strength. The set of simultaneous linear equations are solved such that the normal velocity boundary condition at the midpoint of the segments is satisfied. The total normal velocity at each segment midpoint is zero for inviscid flow. When modeling the effects of the boundary layer however, the normal velocity, $v_{\mathbf{a}}$, is given by the derivative along the surface of the product of the displacement thickness and the tangential velocity, expressed as:

$$
v_{n}=\frac{d\left(u_{e} \delta^{\circ}\right)}{d s}
$$

This surface blowing distribution has the effect of displacing the dividing streamline outward a distance equal to the displacement thickness. Thus, this approach is equivalent to the classic procedure of maintaining the zero normal velocity boundary condition but redefining the 
aerodynamic surface to include the displacement thickness. While the Kutta condition still must be maintained, researchers have found that better results are obtained if it is applied to the displacement surface rather than the original surface.

The boundary layer equations for steady two-dimensional incompressible flows are solved with the velocity distribution at the edge of the boundary layer coming from inviscid flow theory. The total velocity distribution can be expressed in terms of the inviscid velocity and the perturbation velocity due to viscous effects:

$$
u_{e}(x)=u_{e}^{0}(x)+8 u_{e}(x)
$$

where:

$$
\delta u_{e}(x)=\frac{1}{\pi} \int_{x_{e}}^{x_{k}} v_{n} \frac{d \sigma}{x-\sigma}
$$

The range $x_{a} \leq x \leq x_{b}$ is normally taken to be the airfoil plus two chord lengths downstream. Equation 8 provides an outer boundary condition for the viscous flow calculations and represents the interaction between the viscous and inviscid flow. Equation 8 can be generalized to the form:

$$
u_{e}(x)=u_{e}^{x}(x)+\sum_{j=1}^{R} c_{d}\left[\left(u_{e} \delta^{*}\right)_{j}-\left(u_{e} \delta^{*}\right)_{j}^{k}\right]
$$

where $u_{e}{ }^{\alpha}(x)$ is the inviscid velocity distribution containing the displacement thickness effect calculated from the previous sweep. The interaction coefficient matrix, $c_{i j}$, is obtained from a discrete approximation to the Hilbert integral.

The boundary layer solution procedure is derived from Keller's box scheme. The second order finite difference approximations are written in terms of Falkner-Skan variables. Solutions with separation are computed using the inverse form of the equations. The FLARE approximation of Reyhner and Flügge-Lotz ${ }^{13}$ is used which sets the convective term, $u(\partial u / \partial x)$, in the recirculation region to zero. This eliminates the numerical instabilities associated with integrating the boundary layer equation against the direction of local flow. The inaccuracies resulting from this approximation are generally considered small because magnitude of the values of $u$ in the reversed flow region are small compared to the external flow velocity. Although methods exist for improving the numerical method if necessary, no attempt was made to do so here. The finite difference approximations yield a nonlinear system of algebraic equations which are linearized by Newton's method and solved by a block elimination procedure. This procedure is described in more detail in Reference 14. 
In order to deal with surface roughness associated with ice the mixing length expression of the Cebeci-Smith model ${ }^{15}$ has been modified as

$$
L=k(y+\Delta y)\left\{1-e^{\left[-\frac{(y+\Delta y)}{A}\right.}\right\}
$$

where $\Delta y$ is a function of the equivalent sand-grain roughness $k_{\mathbf{p}} . \Delta \mathrm{y}$ can be expressed in terms of dimensionless quantities,

$$
\begin{array}{ll}
\Delta y^{+}=0.9 \sqrt{k_{s}^{+}}-k_{z}^{+} e^{-\left(\frac{k_{i}^{*}}{6}\right)} ; 5 \leq k_{s}^{+} \leq 70 \\
\Delta y^{+}=0.7\left(k_{s}^{+}\right)^{0.58} ; 70 \leq k_{s}^{+} \leq 2000
\end{array}
$$

where

$$
k_{s}^{+}=\frac{k \mu_{\varepsilon}}{v}
$$

and

$$
\Delta y^{+}=\frac{\Delta y u_{\mathrm{r}}}{v}
$$

The roughness is converted into equivalent sand-grain roughness by using the procedure of Smith and Kaups ${ }^{16}$ and assuming the ratio of equivalent sand-grain roughness to the roughness of the applied elements to be a function of the concentration and shape of the roughness elements. More detail on this procedure is given in Reference 3.

Accreted ice on an airfoil can substantially alter the leading edge geometry in a short period of time which causes rapid variations in flow properties. Thus, some difficulty is met trying to obtain acceptable solutions from the inviscid and viscous flow calculations. Thus, "blanketing" and continuation techniques have been employed to minimize this problem. A detailed description of these techniques is given in References 3 and 16.

Work by Shin, et $a^{17}$ has shown that the IBL procedure can acceptably predict drag values of iced airfoils. Results. from that work are shown in Figures 7, 8, and 9. As shown in Figure 
7 , it was found that the IBL procedure had a tendency to underpredict the experimental drag values. This was due in large part to the fact that the theoretical ice shape from which the IBL procedure made its calculations was much smoother than the experimental shape. In any scheme of this nature the quality of the drag predictions will necessarily depend upon the ice shape prediction and the ability to account for roughness. Figures 8 and 9 illustrate that, if the ice shape is given from the experiment, then the IBL procedure closely predicts the experimental values for drag. It should be noted that roughness is an unknown quantity for ice shapes. In order to be physically correct, a roughness (due to ice) should be applied to the ice shape but not to the uniced portion of the airfoil. This roughness value to apply to the ice shape is not known. It has been suggested by Cebeci that a value of no less than 0.002 be used for nondimensional roughness. The method employed here is to use either twice the roughness predicted in Reference 4 or 0.002 , whichever is greater. This roughness value is applied over the first $35 \%$ of the chord of the airfoil and transition to turbulent flow is assumed to occur immediately at the leading edge. As with the ice accretion calculation, the roughness parameter is the major weakness in trying to predict the lift and drag characteristics of an iced airfoil. Much research is needed in order to improve this aspect of the analysis.

\section{Ice Shedding Prediction}

An icing analysis of a rotating system differs from that of a fixed system in that ice shedding becomes a predominant factor. This is because shedding controls the radial extent of ice on the rotor. The radial extent of ice dramatically affects the rotor torque rise. The combination of centrifugal force and vibratory airloads makes shedding commonplace for a helicopter main rotor. In a general sense, ice shedding occurs when the centrifugal, bending, vibratory, and aerodynamic forces acting on a mass of ice causes the stress within the ice to exceed a critical value. When this critical value is surpassed, failure occurs within the ice and aerodynamic forces carry it away.

The shedding model used in the current analysis is an empirical model developed by Flemming. ${ }^{1}$ The Flemming shedding model essentially uses Reference 18 as a pattern. The model predicts the mass and associated centrifugal force for a given radial station. The predicted centrifugal force is then compared to a threshold value. If the centrifugal force exceeds the threshold, then shedding is said to occur. More detail is of the shedding model is given in Reference 1.

Actual determination of the failure stress of the accreted ice is a very difficult task. Scavuzzo, et $a l^{19,20}$ has attempted to experimentally determine the critical shear and normal stresses of accreted ice for various conditions. These results show a strong dependence on surface temperature (above $-11^{\circ} \mathrm{C}$ ) and surface roughness as well as some dependence on wind velocity and droplet size. Historically, as is the case here, these experiments have shown a great deal of scatter in the data. Thus, no method currently exists which will absolutely determine when and where a shedding event will occur. Currently, only probabilities of shedding events can be calculated.

\section{Comparisons}

In late 1989 a heavily instrumented sub-scale model of a generic helicopter main rotor 
was tested in the NASA Lewis Research Center IRT. The rotor consisted of four blades of 1.83 $\mathrm{m}$ diameter and $0.124 \mathrm{~m}$ chord with NACA 0012 cross-section. The model rotor was subjected to a range of icing conditions under various performance states. Data quality was excellent, as indicated in References 12 and 21. The major parameter used for comparison in the present work is rotor torque rise as a function of icing time. Rotor lift loss, although available for comparison, was not examined in detail in this study.

Rotor torque is an integrated value dependent upon the local drag along the rotor blades. It would be desirable to make comparisons to the actual local drag values on the rotor during an icing run. This data is, unfortunately, not available. However, comparisons can be made between the empirical correlation of Flemming and the present analysis. Prior to this, however, it would be appropriate to assess how well the Flemming correlation predicts local drag values. Because of the lack of drag data this can only be done through implication. Figure 10 shows torque rise as a function of icing time for Run 76. Also shown in Figure 10 is the predicted torque rise using the Flemming correlation. Here, the Flemming correlation was incorporated into B65 using a similar method described earlier. More information on the Flemming correlation predictions of the database used here can be found in Reference 21 . It can be seen that there is excellent agreement between the data and the prediction. This particular icing run had a very low rotor speed and thus, no shedding occurred on the rotor. Also, icing extended all the way to the tip of the blades. Therefore, no shedding or icing extent effects are present for this case. Because of this, it can be inferred that the Flemming correlation does a reasonable job of predicting the local drag values. Thus, a comparison between the predicted drag values of the Flemming correlation and the IBL procedure has some merit.

Figures 11 and 12 show comparison between the predicted changes in lift and drag at the $33.8 \%$ radial station. These results (and those in Figures $13-16$ ) are performance maps calculated on the iced rotor corresponding to Run 71 after 20 seconds of icing. It can be seen that, for low Mach numbers, the IBL procedure predicts a higher lift loss than the Flemming correlation. As Mach number increases, however, the IBL procedure begins to underpredict the Flemming results. At the highest Mach number of 0.6 the IBL procedure only predicts $60 \%$ the lift loss that the Flemming correlation calculates. Similar results are seen in Figure 12, where at low Mach numbers there is good agreement between Flemming and the IBL procedure. Again, as Mach number increases the IBL procedure begins to underpredict and finally underpredicts the correlation by about $30 \%$ at a Mach number of 0.6 . Analogous results are seen at radial stations of $66 \%$ and $94.6 \%$ as shown in Figures 13 through 16 . These results are not unlike those found in Reference 17. Again this underprediction is no doubt due to the fact that the IBL procedure uses a very smooth, predicted ice shape rather than the rougher shape found in the experiment. Also, the roughness value for the ice shape has been artificially supplied and the true value is not known.

With these results in mind, it would be expected that the IBL procedure would underpredict torque rise. However, the amount of underprediction is not as dramatic as Figures 11 through 16 might indicate. Typically, a rotor condition will consist of higher flow angles inboard where the Mach number is relatively low, and lower flow angles outboard where the Mach number is high. Thus, in general, local conditions on the rotor will either have low angles of attack or low Mach number. Upon reinspection of Figures 11 through 16 it can be seen that the amount of underprediction is not as significant at low Mach numbers and low 
angles of attack. Results for Run 71 are shown in Figure 17. Here, the predicted torque rise using the IBL procedure is compared to the correlation prediction and the experimental data. First, it can be seen that there is excellent agreement between the correlation and the experiment, as in Figure 10. It can be seen that, unlike Figure 10, significant shedding begins to take place after about 40 seconds of icing. It is at this point that the torque rise begins to level off to a final value of about $40 \%$. The IBL results generally underpredict the experimental torque. It should also be noted that, as icing time increased, the IBL procedure calculations tended to break down for the outer radial stations. This is the region where glaze ice accretion occurs and it is felt that the IBL code had difficulty smoothing the multiple stagnation points. Traditionally, the IBL code has been run by making the performance calculations at the same angle of attack at which the accretion occurs. Here, the iced airfoil is being swept through a range of angles of attack. It is possible that the blanketing procedure currently used in the IBL code may need some work in order to handle this situation. For the cases where calculations did break down, the changes in lift and drag were extrapolated from the inner radial stations. While the correlation generally gives more favorable results than the IBL method for this experiment, the IBL results are encouraging, since the purpose of this effort is to show feasibility of an alternative method. It is hoped that, with some fine tuning in the area of ice roughness, results can be improved. Eventually, aspirations are to make comparisons to full scale flight test data where traditionally, empirical correlations have had difficulty.

\section{Summary}

An alternative method of computing the effects of an icing encounter on the performance of a helicopter main rotor has been discussed. This method makes use of several codes, each of which play a vital part in the overall calculation. The Boeing Helicopters performance code, B65 is used to make the actual performance calculations. A modified version of NASA Lewis's ice accretion code, LEWICE is used to calculate the shape of the accreted ice at various radial locations along the rotor. The IBL procedure of Cebeci determines the effect of the ice accretion on the lift and drag characteristics of two dimensional airfoil slices along the radius. Natural shedding is predicted using an empirical correlation published by Flemming. Torque rise predictions from this jobstream were compared to empirical correlation as well as experimental data. It was found that the jobstream somewhat underpredicted the measured torque rise. It is planned to fine tune the jobstream and make comparisons to full scale experimental data. Since the empirical correlations tend to have difficulty predicting full scale data it is hoped that an analytically based jobstream of this nature can improve the predictions.

\section{References}

${ }^{1}$ Flemming, R.J. and Lednicer, D.A., "High Speed Ice Accretion on Rotorcraft Airfoils," NASA CR-3910, August 1985.

${ }^{2}$ Kwon, O. and Sankar, L., "Numerical Study of the Effects of Icing on the Hover Performance of Rotorcraft," AIAA Paper 91-0663, January 1991. 
${ }^{3}$ Cebeci, T., "Calculation of Flow Over Iced Airfoils," ALA Journal, Vol. 27, 1989, pp. 853-861.

4 Ruff, G.A. and Berkowitz, B.M., "Users Manual for the NASA Lewis Ice Accretion Prediction Code (LEWICE)," NASA CR-185129, May 1990.

s Stepniewski, W.Z. and Keys, C.N., "Blade Element Theory," in Rotary-Wing Aerodynamics. W.Z. Stepniewski, ED. New York, New York: Dover Publications, Inc., 1984, chap. 3, pp. 91-140.

- Johnson, W., "A Comprehensive Analytical Model of Rotorcraft Aerodynamics and Dynamics, Part 1: Analysis Development," NASA TM 81182, June 1980.

7 Hess, J.L. and Smith, A.M.O., "Calculation of Potential Flow About Arbitrary Bodies," in Progress in Aeronautical Sciences. D. Kuchemann, ED. Elmsford, New York: Pergmon Press, 1967, chap. 8, pp. 1-138.

- Frost, W., Chang, H., Shieh, C., and Kimble, K., "Two-Dimensional Particle Trajectory Computer Program," Interim Report for Contract NAS3-22448, 1982.

${ }^{9}$ Messinger, B.L., "Equilibrium Temperature of an Unheated Icing Surface as a Function of Airspeed," Journal of the Aeronautical Sciences, Vol. 20, 1953, pp. 29-42.

${ }^{10}$ Yamaguchi, K. and Hansman, R., "Deterministic Multi-Zone Ice Accretion Modeling," AIAA Paper 91-0265, January 1991.

11 Korkan, K.D., Dadone, L., and Shaw, R.J., "Performance Degradation of Helicopter Rotor Systems in Forward Flight Due to Rime Ice Accretion," AIAA Paper 83-0029, January 1983.

12 Britton, R.K. and Bond, T.H., "A Review of Ice Accretion Data From a Model Rotor Icing Test and Comparison With Theory," AIAA Paper 91-0661, January 1991 (Also, NASA TM-103712, 1991).

${ }^{13}$ Reyner, T.A. and Flügge-Lotz, I., "The Interaction of A Shock Wave With A Laminar Boundary Layer," International Journal of Nonlinear Mechanics, Vol. 3, 1968, pp. 173-179.

${ }^{14}$ Bradshaw, P., Cebeci, T., and Whitelaw, J.H., Engineering Calculation Methods for Turbulent Flows. Academic Press, London, 1981.

15 Cebeci, T. and Smith, A.M.O., Analysis of Turbulent Boundary Layers. Academic Press, New York, 1974. 
16 Smith, A.M.O. and Kaups, K., "Aerodynamics of Surface Roughness and Imperfections," SAE Paper 680198, April 1968.

${ }^{17}$ Shin, J., Berkowitz, B., Chen, H., and Cebeci, T., "Prediction of Ice Shapes and Their Effect on Airfoil Performance," AIAA Paper 91-0264, January 1991.

${ }^{18}$ Loughborough, D.L. and Haas, E.G., "Reduction of the Adhesion of Ice to De-Icer Surfaces," Joumal of the Aeronautical Sciences, Vol. 13, No. 3, March 1946.

19 Scavuzzo, R.J., Chu, M.L., and Ananthaswamy, V., "Influence of Aerodynamic Forces in Ice Shedding," AIAA Paper 91-0664, January 1991.

${ }^{20}$ Scavuzzo, R.J. and Chu, M.L., "Structural Properties of Impact Ices Accreted on Aircraft Structures," NASA CR 179580, January 1987.

${ }^{21}$ Flemming, R.J. and Saccullo, A., "Tests of a Model Main Rotor in the NASA Lewis Research Center Icing Research Tunnel," NASA CR 189071, December 1991. 


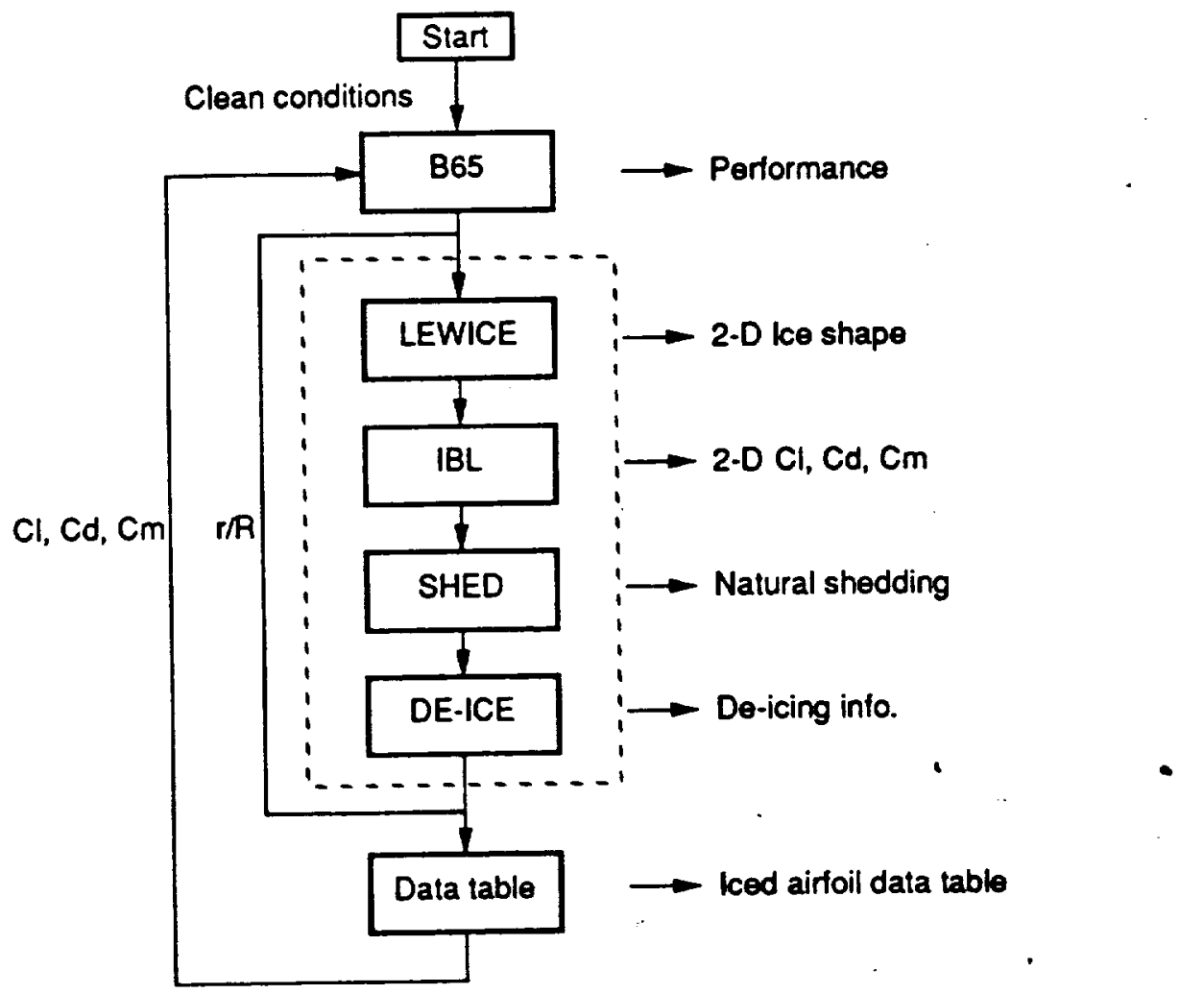

Figure 1. Helicopter Performance In Icing Prediction Jobstream.

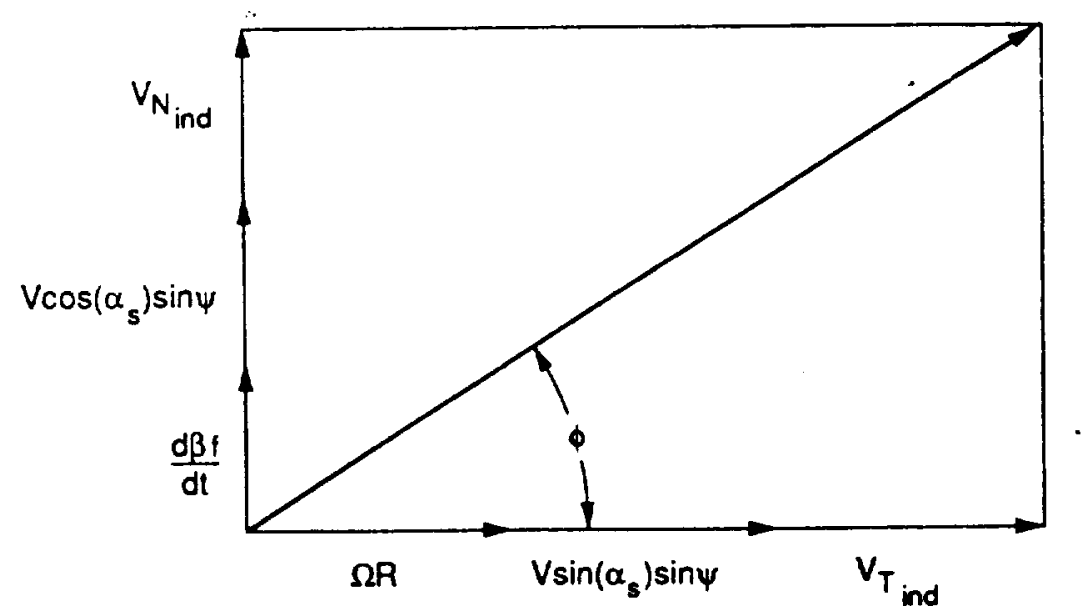

Figure 2. Blade section velocity triangle. (Ref. 6) 


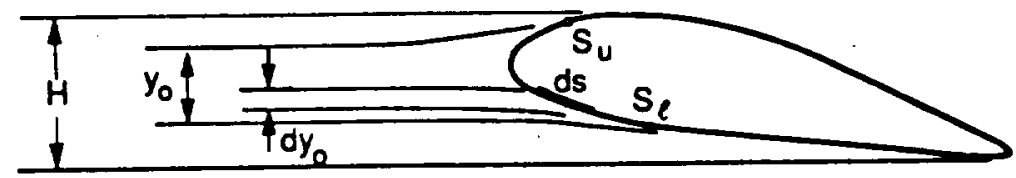

$S_{U}=$ Upper - surface impingement limit

$S_{\ell}=$ Lower - surface impingement limit

$H=$ Forward projection of the airfoil height

Total collection efficiency

$$
\begin{aligned}
E_{m} & =\frac{y_{0}}{H} \\
E_{m} & =\frac{1}{H} \int_{S_{l}}^{S_{u}} \beta d s \\
\text { Total collection efficiency } \quad \beta & =\frac{d y_{0}}{d s}
\end{aligned}
$$

Figure 3. Definition of total and local collection efficiency. (Ref.

4)

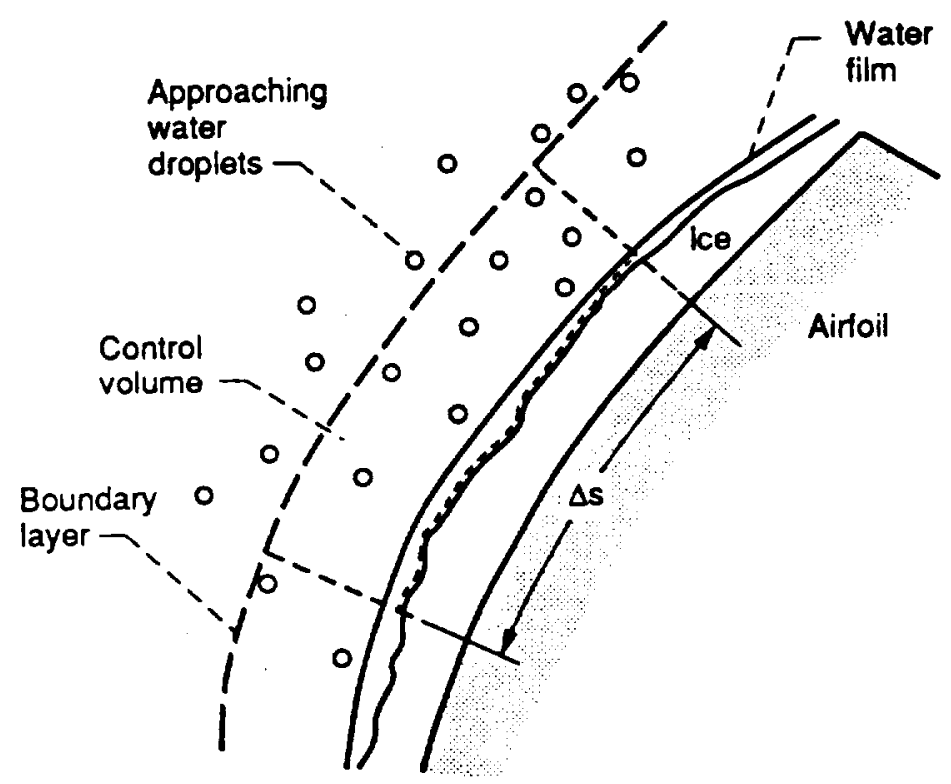

Figure 4. Control volume on the icing surface. (Ref. 4) 


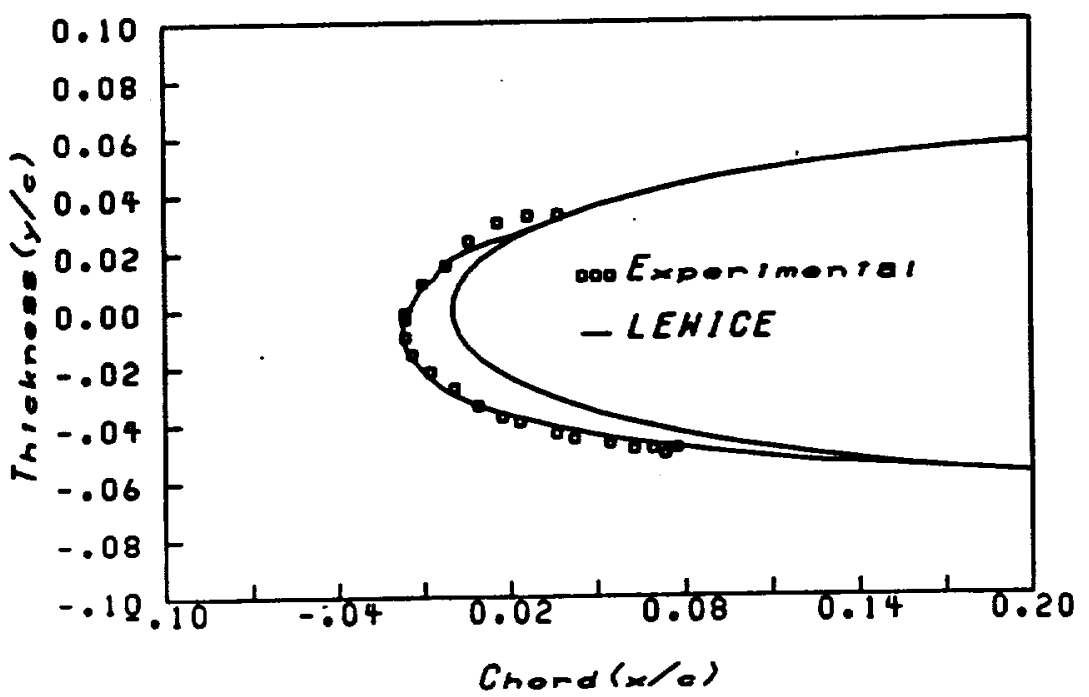

Figure 5. Comparison between experiment and theoretical prediction of LEWICE. (Ref. 12)

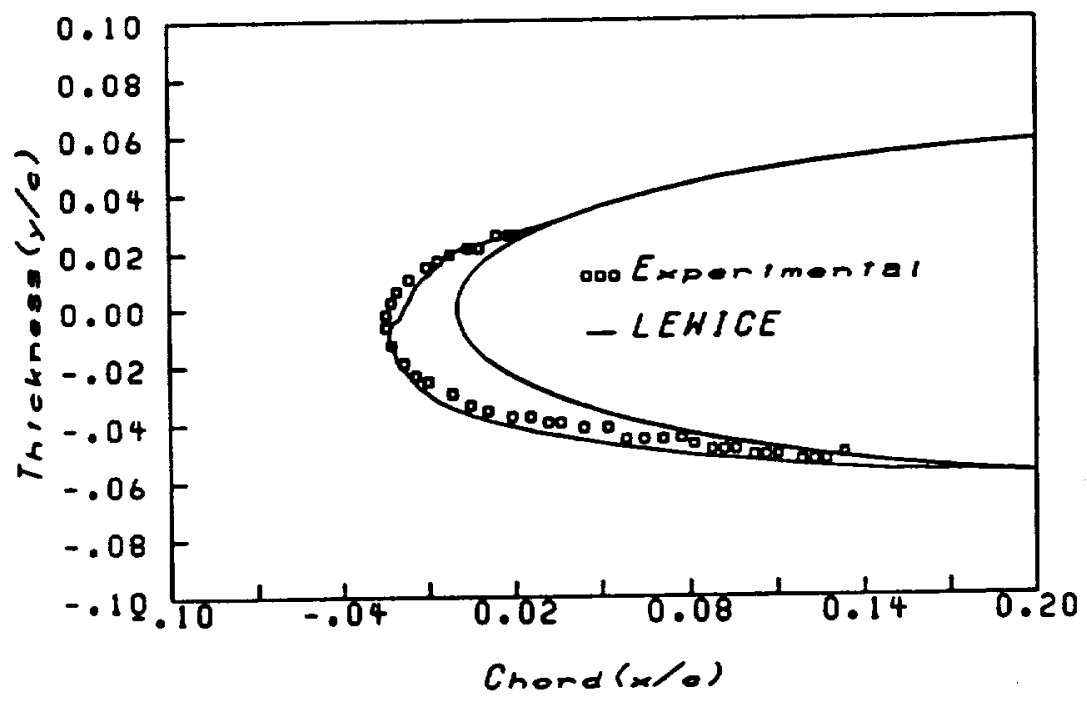

Figure 6. Comparison between experiment and theoretical prediction of LEWICE. (Ref. 12) 


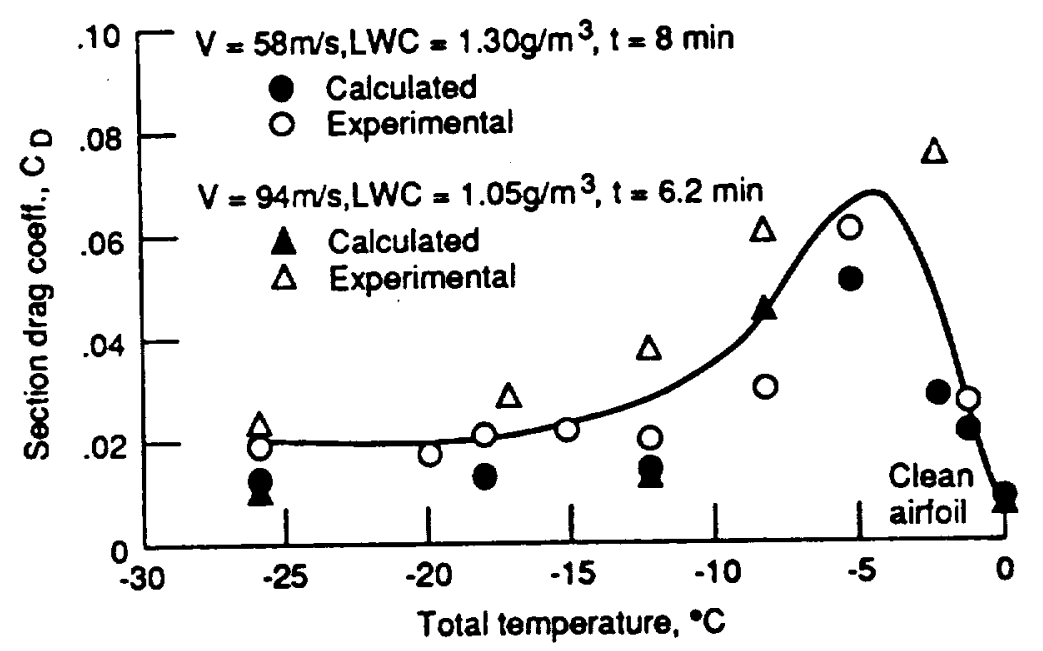

Figure 7. Comparison between experiment and theoretical prediction of IBL procedure. (Line is a fit for experimental data.) (Ref. 17)

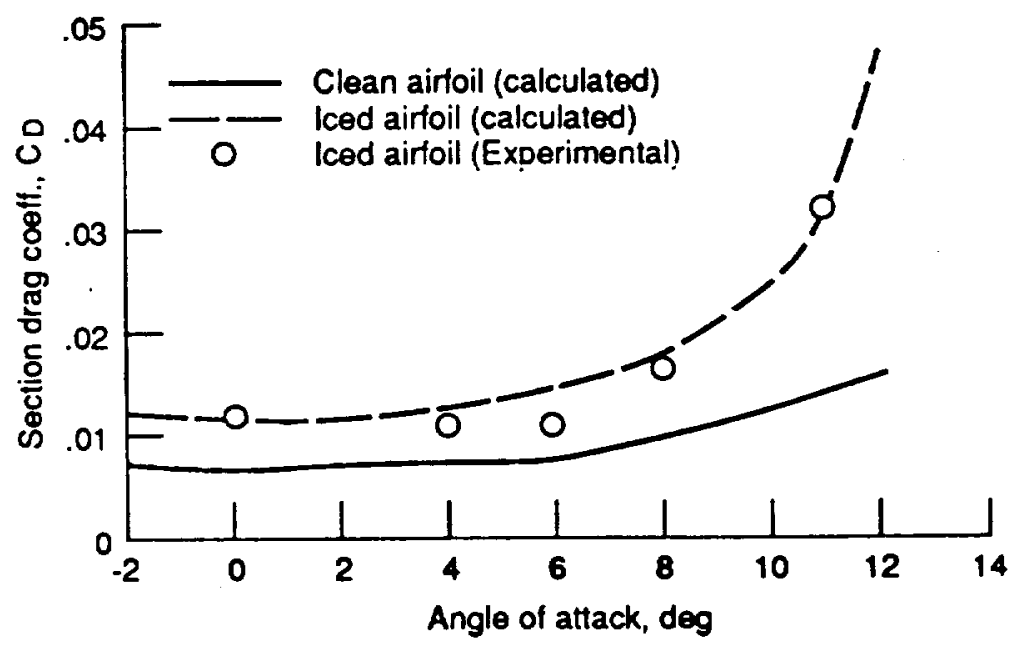

Figure 8. Comparison between experiment and theoretical prediction of IBL procedure. $\left(\phi=4^{\circ}, \mathrm{V}=58 \mathrm{~m} / \mathrm{sec}, \mathrm{T}_{1}=-27.8\right.$ ${ }^{\circ} \mathrm{C}, \mathrm{LWC}=1.0 \mathrm{~g} / \mathrm{m}^{3}, \mathrm{MVD}=12 \mu \mathrm{m}, \tau=5 \mathrm{~min}$.) (Ref. 17) 


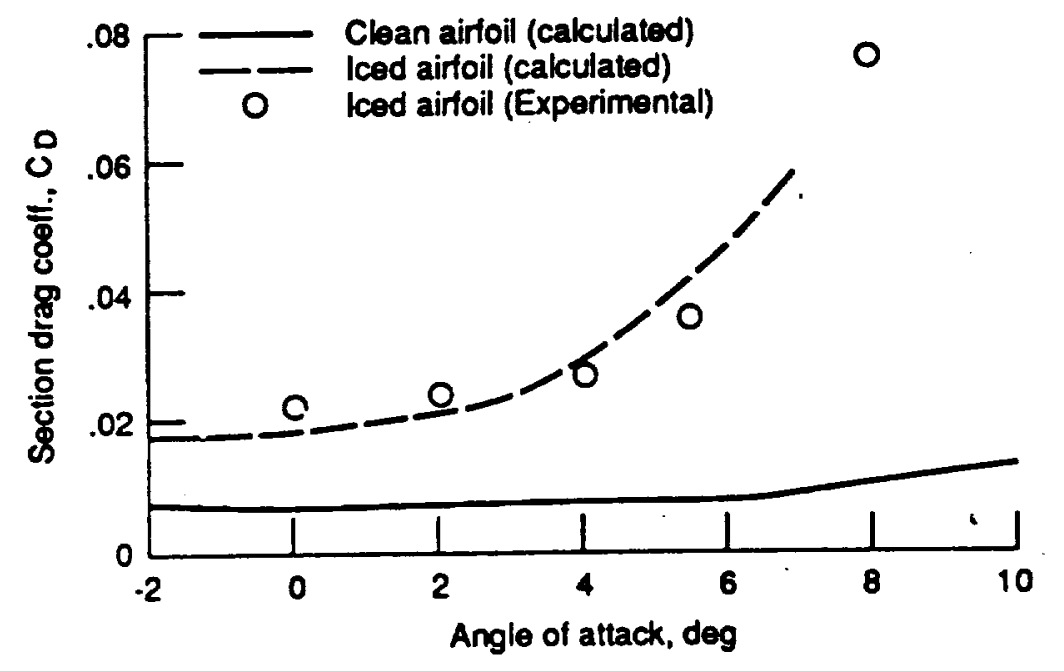

Figure 9. Comparison between experiment and theoretical prediction of IBL procedure. $\left(\phi=4^{\circ}, \mathrm{V}=58 \mathrm{~m} / \mathrm{sec}, \mathrm{T}_{\mathrm{s}}=\right.$ $9.67{ }^{\circ} \mathrm{C}, \mathrm{LWC}=1.3 \mathrm{~g} / \mathrm{m}^{3}, \mathrm{MVD}=20 \mu \mathrm{m}, \tau=5 \mathrm{~min}$.) (Ref. 17)

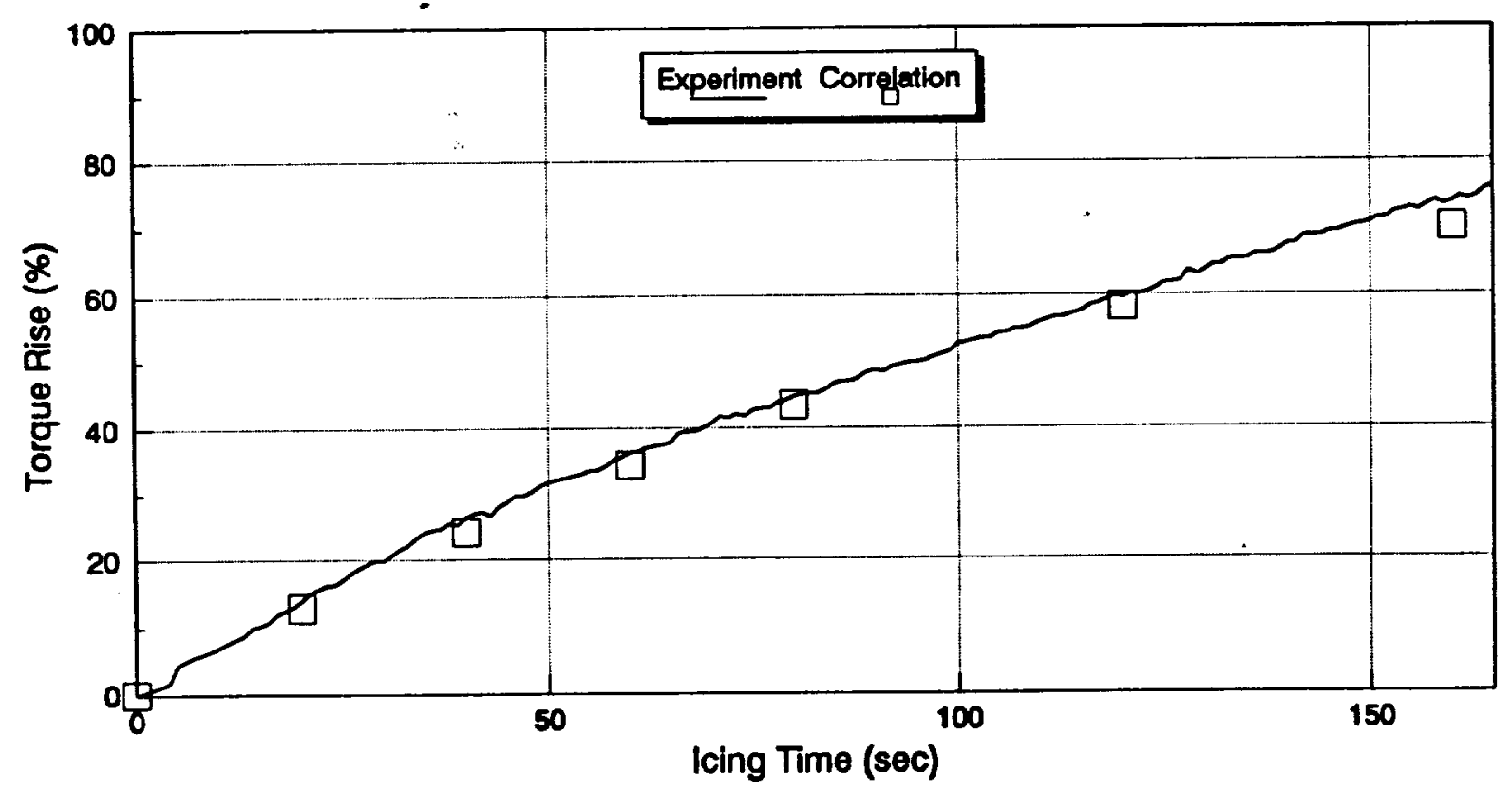

Figure 10. Comparison between experimental data and correlation prediction for Run 76 (LWC $=0.75 \mathrm{~g} / \mathrm{m}^{3}, \mathrm{MVD}=15 \mu \mathrm{m}, \mu=$ $0.306, \mathrm{\Omega R}=119.8 \mathrm{~m} / \mathrm{s}, \mathrm{C}_{\mathrm{L}} / \sigma=0.0645, \mathrm{~T}_{\mathrm{s}}=-15^{\circ} \mathrm{C}$ ). 


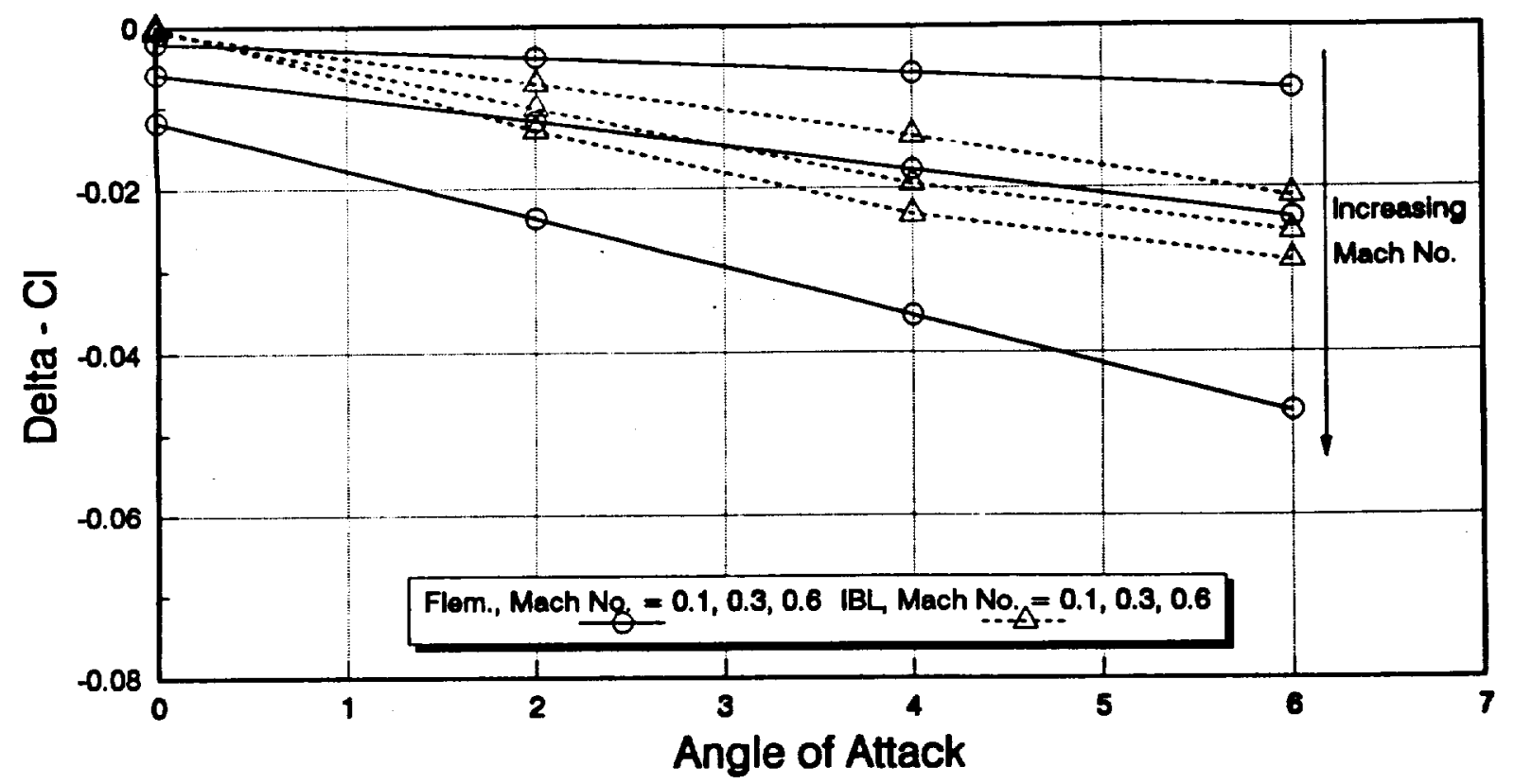

Figure 11. Comparison of $\Delta C_{1}$ predictions for $r / R=0.338, \Delta \tau$ $=20 \mathrm{sec}$., Run 71 (LWC $=0.5 \mathrm{~g} / \mathrm{m}^{3}, \mathrm{MVD}=15 \mu \mathrm{m}, \mu=$ $0.197, \mathrm{RR}=205.7 \mathrm{~m} / \mathrm{s}, \mathrm{C}_{\mathrm{L}} / \sigma=0.064, \mathrm{~T}_{\mathbf{1}}=-15^{\circ} \mathrm{C}$ ).

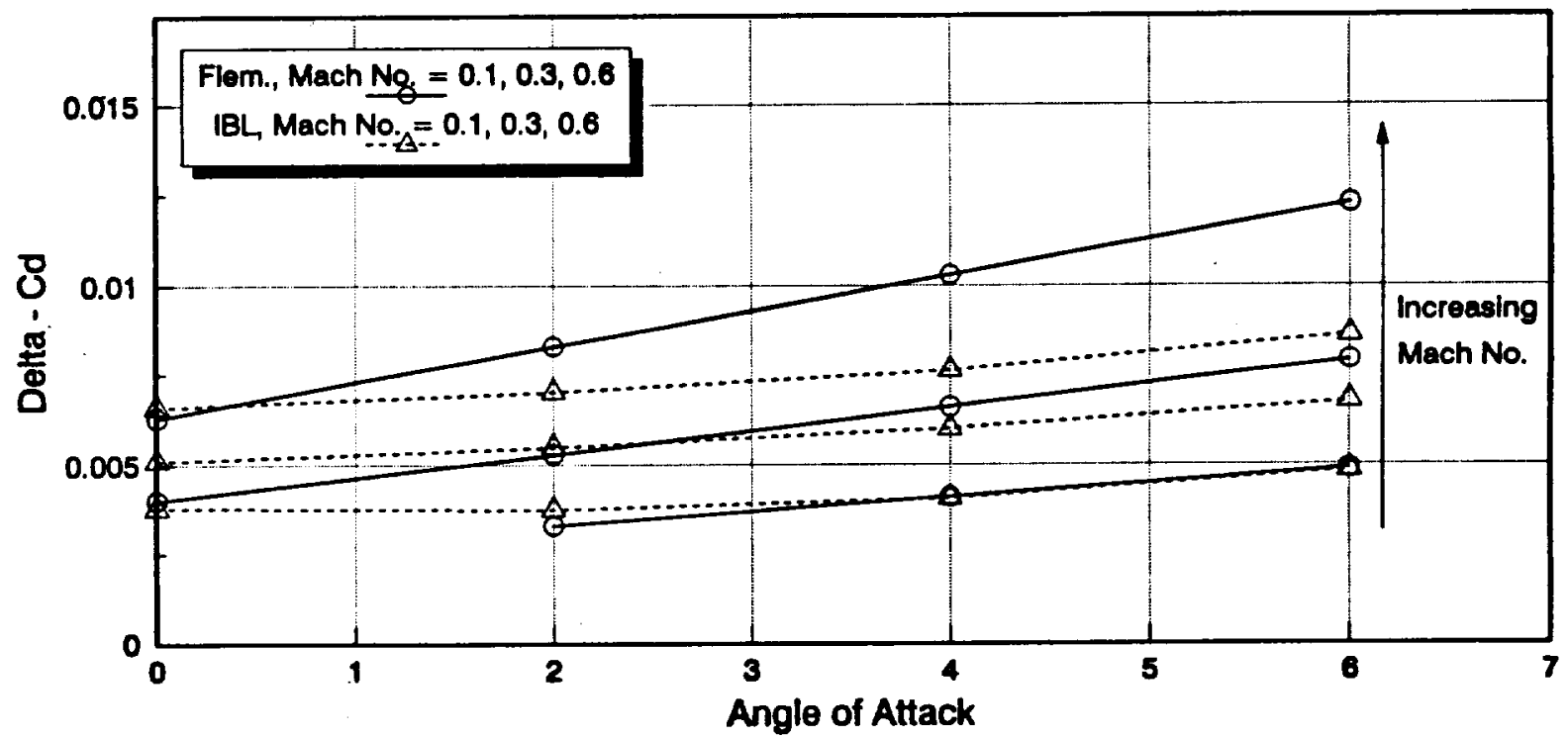

Figure 12. Comparison of $\Delta C_{d}$ predictions for $r / R=0.338, \Delta \tau$ $=20 \mathrm{sec}$, Run 71 (LWC $=0.5 \mathrm{~g} / \mathrm{m}^{3}, \mathrm{MVD}=15 \mu \mathrm{m}, \mu=$ $\left.0.197, \mathrm{NR}=205.7 \mathrm{~m} / \mathrm{s}, \mathrm{C}_{\mathrm{L}} / \sigma=0.064, \mathrm{~T}_{\mathrm{a}}=-15^{\circ} \mathrm{C}\right)$. 


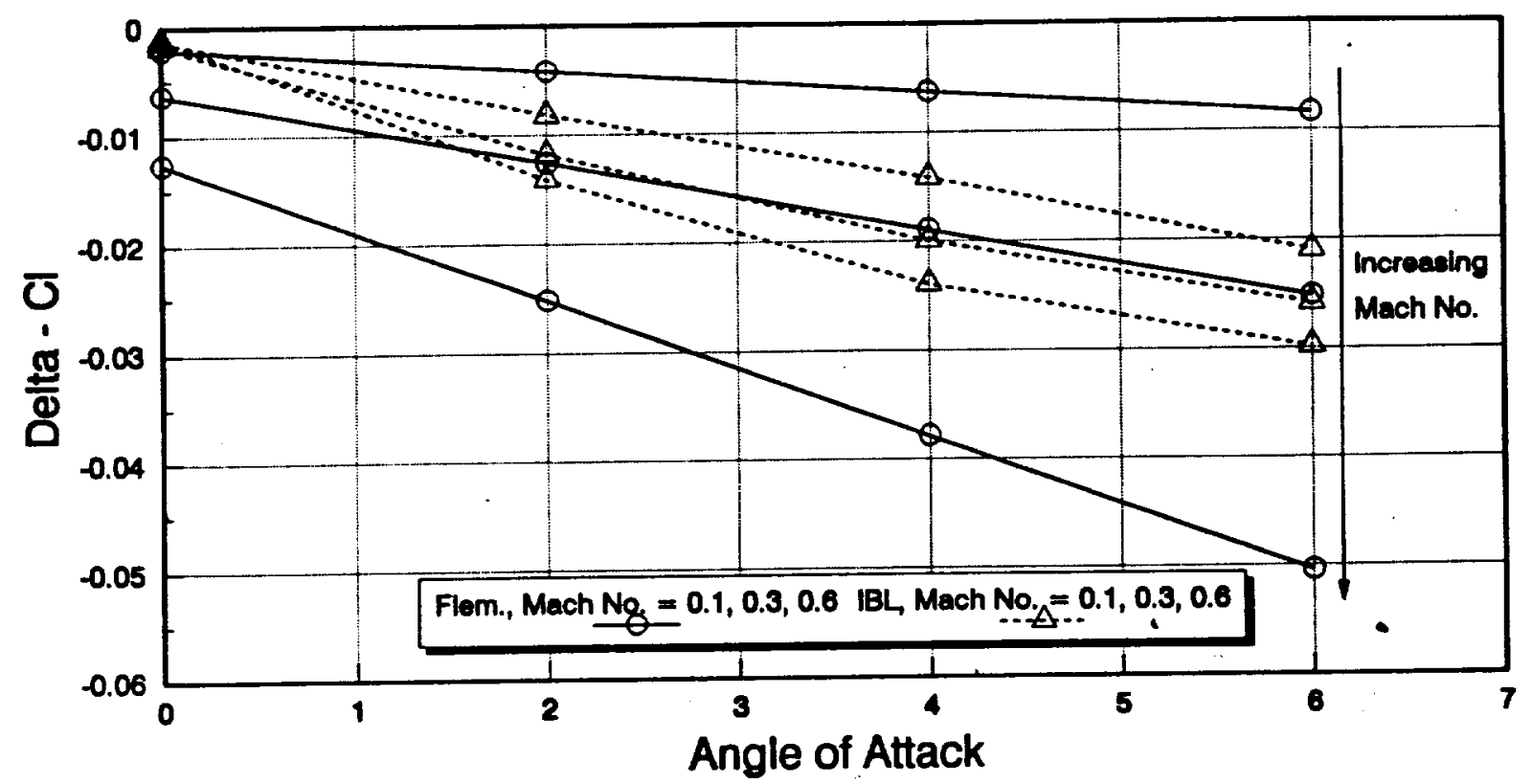

Figure 13. Comparison of $\Delta C_{1}$ predictions for $r / R=0.660, \Delta \tau$ $=20$ sec., Run 71 (LWC $=0.5 \mathrm{~g} / \mathrm{m}^{3}$, MVD $=15 \mu \mathrm{m}, \mu=$ $0.197, \mathrm{RR}=205.7 \mathrm{~m} / \mathrm{s}, \mathrm{C}_{\mathrm{L}} / \sigma=0.064, \mathrm{~T}_{\mathrm{v}}=-15^{\circ} \mathrm{C}$ ).

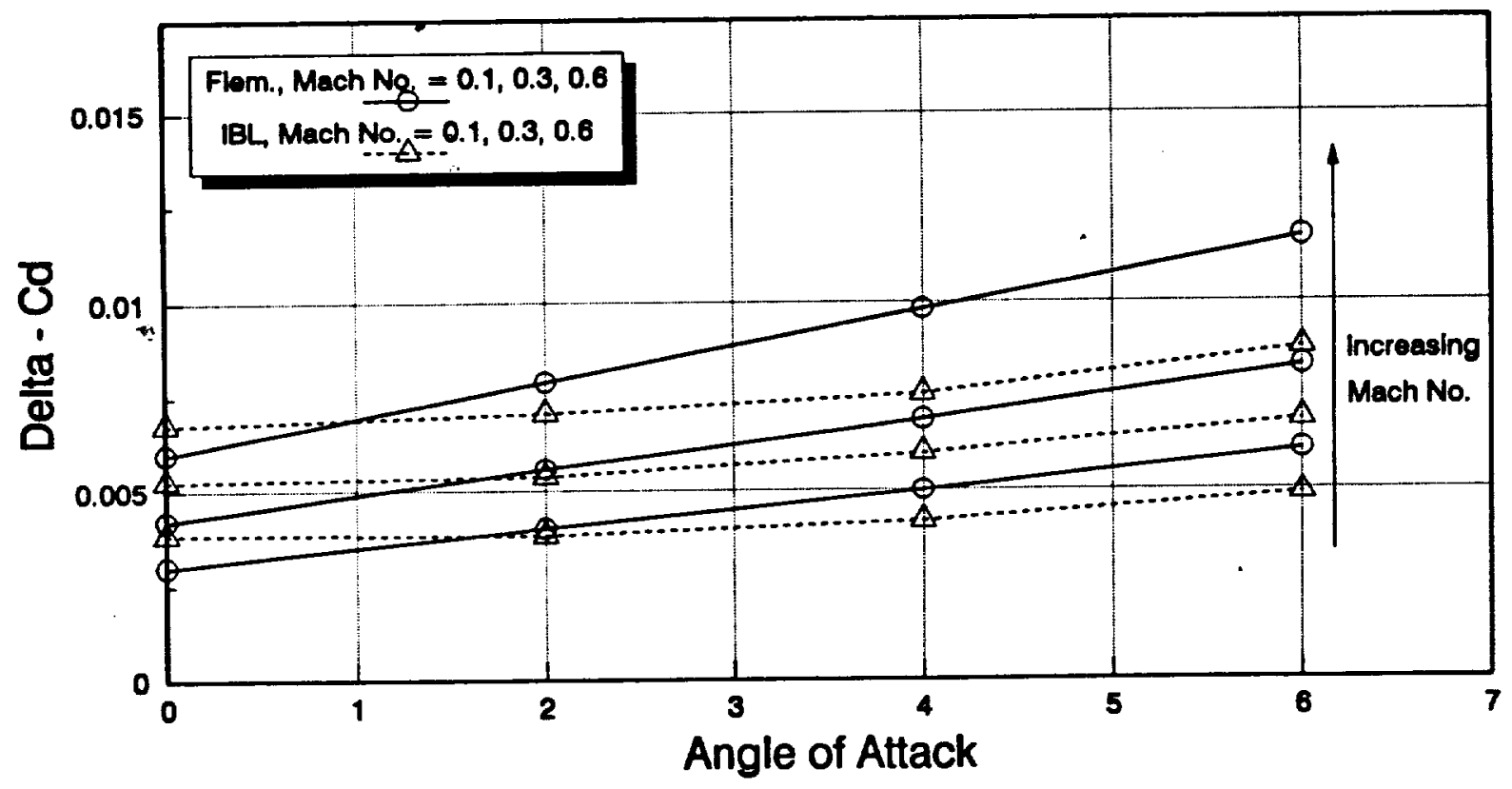

Figure 14. Comparison of $\Delta C_{d}$ predictions for $r / R=0.660, \Delta \tau$ $=20 \mathrm{sec}$., Run 71 (LWC $=0.5 \mathrm{~g} / \mathrm{m}^{3}, \mathrm{MVD}=15 \mu \mathrm{m}, \mu=$ $0.197, \mathrm{RR}=205.7 \mathrm{~m} / \mathrm{s}, \mathrm{C}_{\mathrm{l}} / \sigma \doteq 0.064, \mathrm{~T}_{\mathrm{s}}=-15^{\circ} \mathrm{C}$ ). 


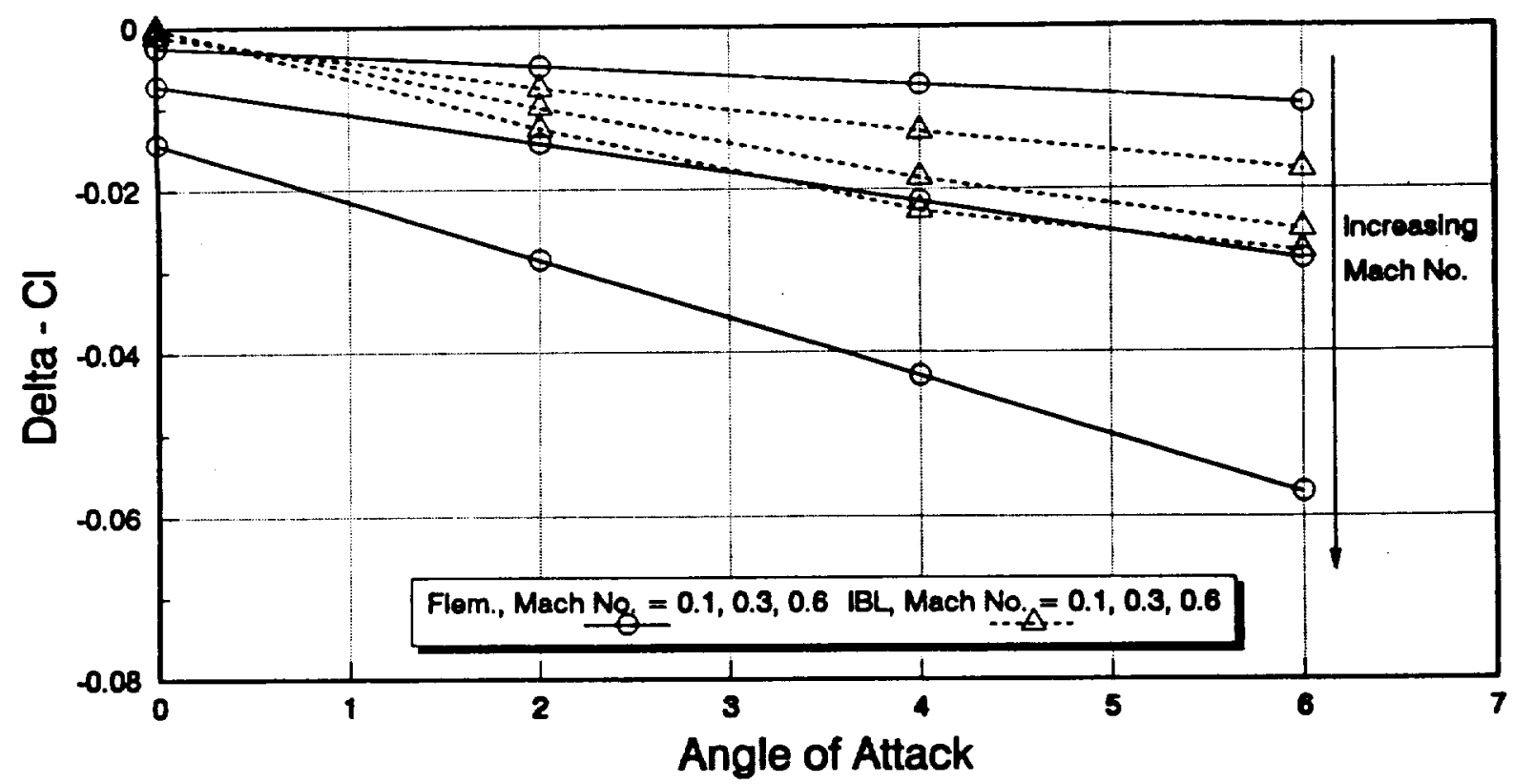

Figure 15. Comparison of $\Delta C_{1}$ predictions for $r / R=0.946, \Delta \tau$ $=20$ sec., Run 71 (LWC $=0.5 \mathrm{~g} / \mathrm{m}^{3}, \mathrm{MVD}=15 \mu \mathrm{m}, \mu=$ $\left.0.197, \mathrm{RR}=205.7 \mathrm{~m} / \mathrm{s}, \mathrm{C}_{\mathrm{L}} / \sigma=0.064, \mathrm{~T}_{\mathrm{a}}^{\circ}=-15^{\circ} \mathrm{C}\right)$.

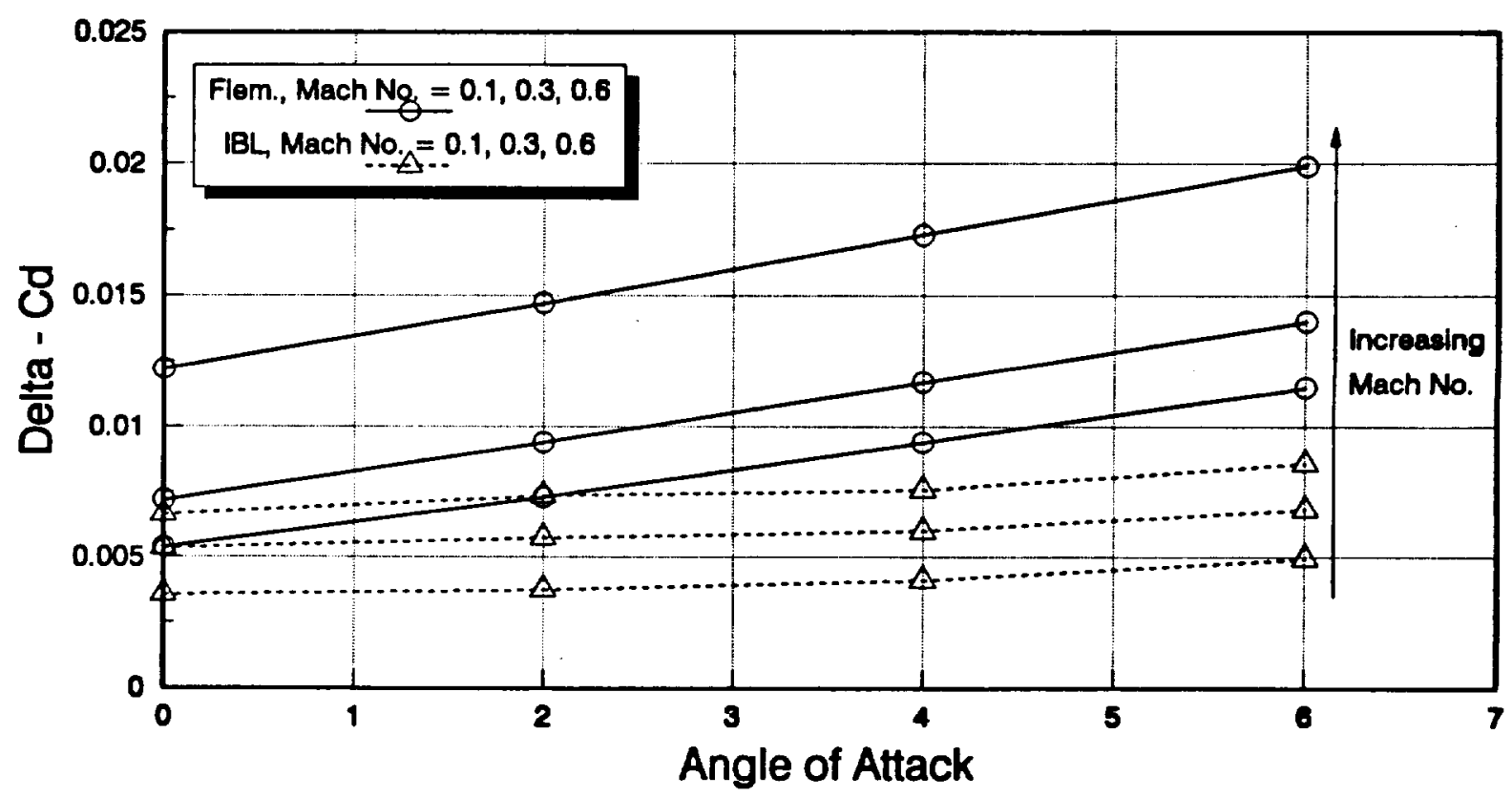

Figure 16. Comparison of $\Delta C_{d}$ predictions for $\mathrm{r} / \mathrm{R}=0.946, \Delta \tau$ $=20$ sec., Run 71 (LWC $=0.5 \mathrm{~g} / \mathrm{m}^{3}, \mathrm{MVD}=15 \mu \mathrm{m}, \mu=$ $0.197, \mathrm{RR}=205.7 \mathrm{~m} / \mathrm{s}, \mathrm{C}_{\mathrm{L}} / \sigma=0.064, \mathrm{~T}_{\mathrm{s}}=-15^{\circ} \mathrm{C}$ ). 


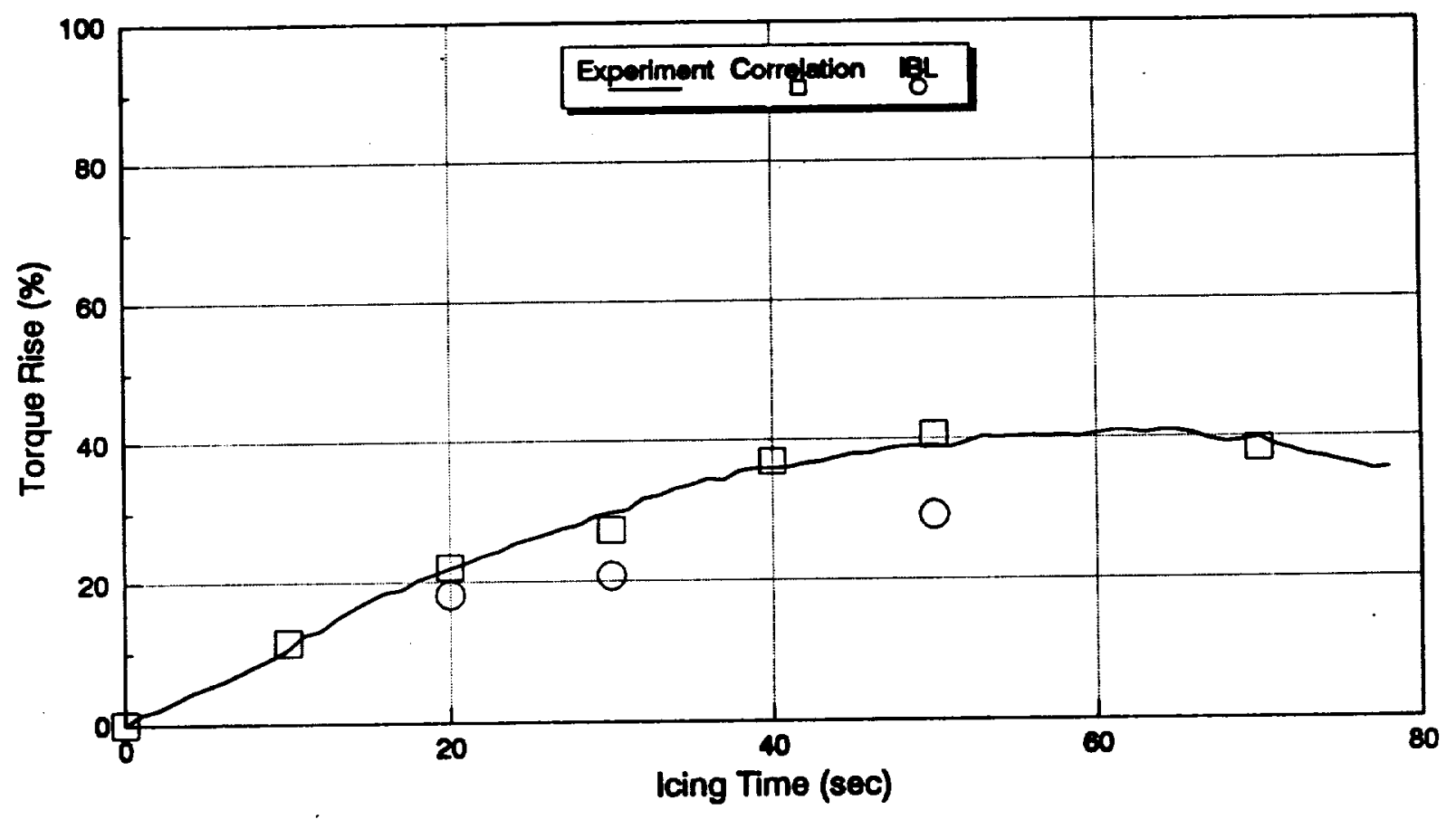

Figure 17. Comparison of rotor torque predictions for Run 71

(LWC $=0.5 \mathrm{~g} / \mathrm{m}^{3}, \mathrm{MVD}=15 \mu \mathrm{m}, \mu=0.197, \mathrm{RR}=205.7$ $\left.\mathrm{m} / \mathrm{s}, \mathrm{C}_{\mathrm{L}} / \sigma=0.064, \mathrm{~T}_{1}=-15^{\circ} \mathrm{C}\right)$. 


.


Public reporting burden for this collection of information is extimaled to average 1 hour per responce, including the time for reviowing hatructions, eearching existing data cources, gathering and maintaining the dala needed, and completing and reviewing the colloction of information. Send comments reparding this burden eetimate or amy ofter aspact of this collection of Information, including supgestions for reducing this bunden, to Washington Headquarters Services, Directorats lor intormation Operations and Peports, 1215 Jellenon Davis Highway, Sulte 1204, Alington, VA 22202-4302, and to the Otfice of Management and Budget, Papenwork Reduction Project (0704-0188), Waehingion, DC 20503.

\begin{tabular}{|l|c|c|}
\hline 1. ACENCY USE ONLY (Leave bJank) & $\begin{array}{c}\text { 2. REPORT DATE } \\
\text { January } 1992\end{array}$ & $\begin{array}{r}\text { 3. REPORT TYPE AND DATES COVERED } \\
\text { Final Contractor Report }\end{array}$
\end{tabular}

\section{TIRLE AND SUBTTLE}

Development of an Analytical Method to Predict Helicopter Main Rotor Performance in Icing Conditions

8. FUNDING NUMBERs

6. AUTHOR(8)

WU-505-68-10

Randall K. Britton

\section{PERFORMING ORGANIZATION NAME(S) AND ADDRE8S(ES)}

Sverdrup Technology, Inc.

Lewis Research Center Group

2001 Aerospace Parkway

Brook Park, Ohio 44142

9. SPONSORING/MONITORING AGENCY NAMES(S) AND ADDRESS(ES)

National Aeronautics and Space Administration

Lewis Research Center

Cleveland, Ohio 44135-3191
0. Performino oroanization

REPORT NUMBER

E-6779

10. SPONSORINOMONITOAINO AOENCY REPOAT NUMBER

NंASA CR -189110

AIAA-92-0418

\section{SUPPLEMENTARY NOTE8}

Project Manager, John J. Reinmann, Propulsion System Division, NASA Lewis Research Center. Prepared for the 30th

Aerospace Sciences Meeting and Exhibit sponsored by the American Institute of Aeronautics and Astronautics, Reno,

Nevada, January 6-9, 1992. Responsible person, Randall K. Britton, (216) 826-2237.

12.. DISTRIBUTIONJAVAILABILITY STATEMENT

12b. DISTAIBUTION CODE

Unclassified - Unlimited

Subject Category 02

13. ABSTRACT (Max/mum 200 words)

Historically, certification of a helicopter for flight into known icing conditions has been a problem. This is because of the current emphasis on flight testing for verification of system performance. Flight testing in icing conditions is difficult because, in addition to being dangerous and expensive many times conditions which are sought after cannox be readily found in nature. The problem is compounded for helicopters because of their amall range in comparison to many fixed wing aircraft. Thus, helicopters are forced to wait for conditions to occur in a certain region rather than seeking them out. These and other drawbacks to flight testing have prompted extreme interest in developing validated alternatives to flight testing. One such alternative is theoretical prediction. It is desirable to have the ability to predict how a helicopter will perform when subjected to icing conditions. A problem such as this is very complex and requires several steps of calculation. For the purpose of this study calculations will be restricted to the main rotor. The flowchart given Figure 1 demonstrates the steps necessary for such a calculation. Initially, the clean performance of the helicopter is required. The computational 10ol used in this study to obtain performance is the lifting line analysis of B65 developed by Boeing Helicopters. B65, as do most andyses of this type incorporates experimental data into data banks in order to determine the section lift, dag, and moment characteriatics of various airfoils at different Mach numbers and angles of atuck. The local flow angle is calculated at user specified radial locations. This flow angle, along with the local Mach number is then cross referenced with the airfoil tubles to obtain the local section characteristics. The local characteristics are then integrated together to obtain the entire rotor atuributes. Once the clean performance is koown, characterizstion of the type and shape of ice which accretes on the rotor blades is obtained using the analysis of LEWICE ${ }^{\prime}$ The Interactive Boundary Layer (IBL) method developed by Cebeci $^{2}$ then calculates the 2 dimensional characteristics of the of the iced airfoil for input into the airfoil duta banks of B65. Both LEWICE and IBL are 2 dimensional codes and thus several calculations along the radius blade are required to characterize the entire iced rotor blade. A complete analysis would also require calculation of any natural shedding events as well as de-icing. For the purpose of this study however, calculations are restricted to natural shedding and it is assumed that no de-icing take place. Once the new lift, drag, and moment characteristics are known for the entire blade radius this information is fed into B65 where the iced performance is then calculated. Dats taken from the NASA Lewis model rotor icing test program is used as a data base for comparison. Comparisons are also made between this method and the more traditional method besed on empirical correlation. ${ }^{3}$ Conclusions are drawn as to how well results compare with experiment. Limitations of this type of jobstream are pointed out and recomrnendations are made for future improvements.

14. SUBJECT TERMS

Helicopter icing; Performance prediction

15. NUMBER OF PAGES 26

16. PRICE CODE

17. SECURITY CLASSIFICATION OF REPORT

Unclassified

18. SECURITY CLASSIFICATION
OF THIS PAOE
Unclassified

Unclassified
19. SECURITY CLASSIFICATION OF ABSTAACT Unclassified 